\title{
LogoTakt - hochfrequente, getaktete Belieferung in kleinen Sendungseinheiten
}

\author{
Dipl.-Wi.-Ing. Judith Weiblen, Dipl.-Wi.-Ing. Heiko Breier, \\ Prof. Dr.-Ing. Kai Furmans \\ KIT - Karlsruher Institut für Technologie \\ Institut für Fördertechnik und Logistiksysteme
}

Abstract: Innerhalb des im Rahmen des Projektes LogoTakt (BMWi, Förderkennzeichen $19 \quad$ G $7036 \quad$ E) entwickelten Transportkonzeptes für Großladungsträger wird zwischen Versendern und Empfängern eine hochfrequente Taktung eingeführt, die zu einer Reduktion der Bestände und höherer Zuverlässigkeit führt. Um zu vermeiden, dass durch die höhere Frequenz die Transportkosten steigen, müssen die Transporte in einem offenen Netzwerk mehrerer Teilnehmer stattfinden und die so entstehenden Konsolidierungseffekte genutzt werden. Im Projekt LogoTakt wurde für ein solches Netzwerk ein Anreiz- und Vergütungsmodell entwickelt, das die Zusammenarbeit von Versendern, Verladern, Spediteuren und Empfängern regelt. Durch die erhöhte Zuverlässigkeit entsteht trotz höherer Frequenz weniger Dispositionsaufwand, da die Transporte auf strategischer Ebene geplant werden können. Weiterhin wird die Disposition durch ein Abweichungsmanagement untersützt, das alle laufenden Transporte überwacht und bei Bedarf aus der Historie mögliche Lösungen für die Abweichung vorschlägt. Diese KI-Komponente sorgt gleichzeitig durch eine ständige Analyse der historischen Daten für einen strukturierten kontinuierlichen Verbesserungsprozess, der die operative mit der strategischen Ebene verbindet. LogoTakt ermöglicht durch die Integration strategischer und operativer Komponenten zu einem Softwaretool eine bessere Planbarkeit eines komplexen Transportsystems unter Einbeziehung von Lean-Erkenntnissen. Letztendlich können durch dieses Konzept gleichzeitig Ziele, die häufig im Bezug auf Green Logitics diskutiert werden, wie beispielsweise $\mathrm{CO}^{2}$ Einsparungen oder die Reduktion von Straßenkilometern, erreicht werden.

\section{Motivation}

Für Deutschland bieten sich durch den Ausbau zur logistischen Drehscheibe Europas für innereuropäische Verkehre (vgl. Abbildung 1) erhebliche Wachstumschancen auf dem Markt für logistische Leistungen, insbesondere im Transport- und Umschlagbereich. Die Wachstumschancen können jedoch nur genutzt werden, wenn es vor dem Hintergrund der bereits heute ausgelasteten Kapazitäten im Straßenverkehr gelingt, Verkehr von der Straße auf andere Transportmedien wie z.B. die Bahn zu verlagern (vgl. [Car08]). Die Transportleistung im Straßengüterverkehr hat sich seit der Wiedervereinigung Deutschlands (1991) verdoppelt und ist somit durchschnittlich um 3,9 Prozent pro Jahr gestiegen [Rat10]. 


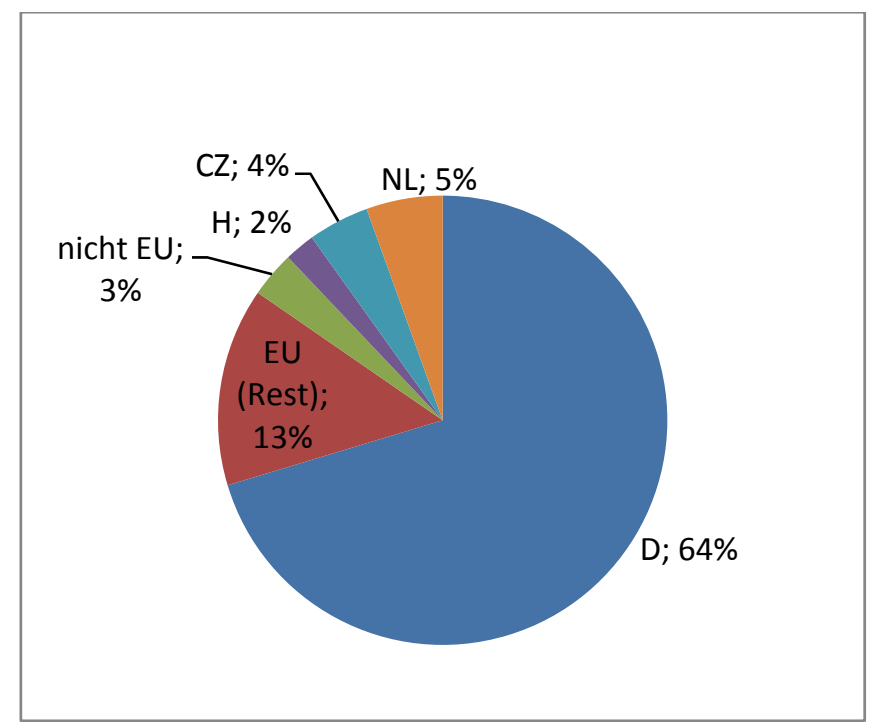

Abbildung 1 Anteil der Fahrleistung nach Nationalität im 1. Halbjahr 2010, Quelle: [Bun10]

Durch jüngere Entwicklungen in der Produktions- und Distributionslogistik gibt es neue Möglichkeiten zur Verlagerung dieses Aufkommens. Moderne Konzepte in diesen Bereichen vermeiden es, durch kurzfristige Ereignisse getrieben zu werden, weil dies zu starken Fluktuationen im Produktions- und Transportaufkommen führen kann. Sie sehen vielmehr vor, Produktion und Beschaffung im Gesamtvolumen zu nivellieren und in Taktprozessen zu betreiben.

Aufgrund des allgemeinen Trends hin zur Globalisierung und damit auch zur Expansion in Unternehmen werden vermehrt freie Mittel in Form von Cash Flows benötigt. Um einen solchen Mittelüberschuss zu erzielen, können beispielsweise gebundene Mittel in Form von Beständen abgebaut werden, allerdings darf dadurch die Versorgung der Produktion nicht gefährdet werden.

Bei Einführung von Lean Production können derzeit zwar Bestände zwischen den einzelnen Produktionsschritten gesenkt werden, zur Sicherung der Versorgung müssen jedoch Bestände im Wareneingang erhalten bleiben bzw. aufgrund der Konsolidierung von Transporten am Warenausgang sogar vergrößert werden. In Abbildung 2 ist exemplarisch der Bestandverlauf vor der Durchführung eines Nivellierungsprojektes, und 5 Jahre nach der erfolgreichen Umsetzung der Nivellierung in einem Unternehmen dargestellt. Deutlich zu erkennen ist, dass sich Bestände zwar innerhalb der Produktion reduziert haben, gleichzeitig aber eine Anhäufung von Beständen an den Unternehmensschnittstellen (Warenein- und ausgang) stattgefunden hat. Mithilfe des Transportkonzeptes LogoTakt können diese Bestände jedoch durch einen höherfrequenten und robusten Transport ebenfalls abgebaut, und damit Cash Flows freigesetzt werden. 


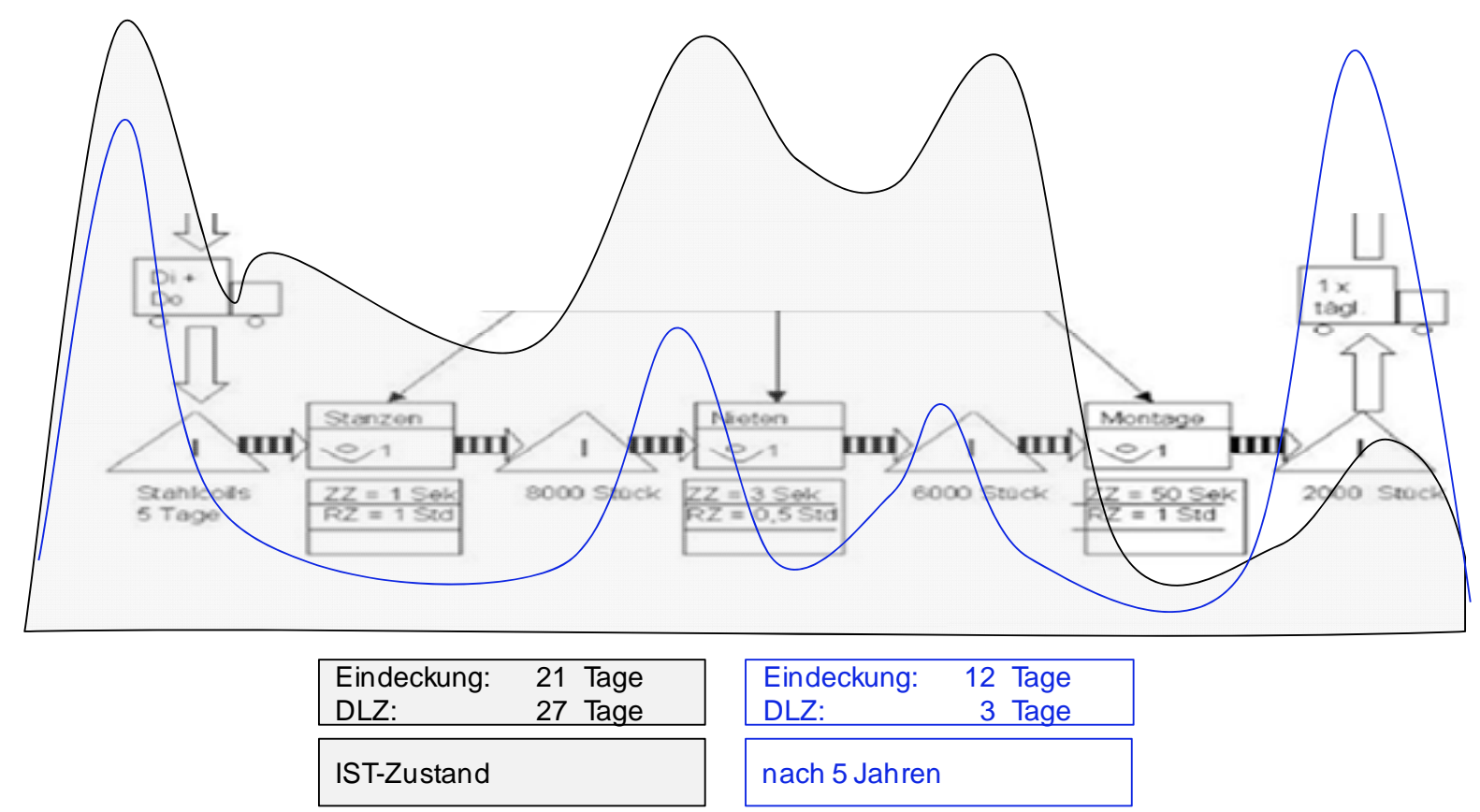

Abbildung 2 beispielhafte Bestandsentwicklung bei Einführung von Lean Production, Quelle: IFL

\section{Funktionsweise von LogoTakt}

Der Grundgedanke von LogoTakt ist es, in so hoher Frequenz anzuliefern, dass eine direkte Belieferung beispielsweise von Hallensupermärkten ohne erneute Lagerung möglich werden kann. Dadurch können vor allem die zur Konsolidierung von Transporten gebildeten Bestände im Warenein- und Warenausgang abgebaut werden (vgl. Abbildung 2, [Bre09]).

Um externe Handlingstufen zu überspringen und teure Zwischenlagerung $\mathrm{zu}$ vermeiden, muss sich die Taktfrequenz über die gesamte Lieferkette bis zu den Zulieferern fortsetzen [Pul10a]. Deswegen konzentriert sich LogoTakt auf eine Erhöhung der Transportfrequenz auf allen Transportrelationen und damit auch eine Steigerung der Abholfrequenz bei den Zulieferern. Die Erhöhung der Frequenz trägt gleichzeitig zur Verringerung des Bullwhip Effekts bei und reduziert Schwankungen im Transportaufkommen, die durch große Losgrößen entstehen. Hohe Frequenz zieht zwangsläufig kleinteiligere Transporte nach sich [Fur10].

Kleinteilige Transporte können nur wirtschaftlich bewältigt werden, wenn es auf allen Transportwegen zu einer Bündelung bzw. Konsolidierung der Transportströme kommt, Transportkapazitäten also durch Zusammenfassen von Transporteinheiten aus unterschiedlichen Quellen und mit unterschiedlichen Senken möglichst gut ausgenutzt werden [Hin09]. LogoTakt ist daher als offenes Netz von Transporteuren konzipiert, in dem Transporte auch transporteurübergreifend geplant werden können [Oel09].

Als Schwerpunkt hat sich LogoTakt dabei vorgenommen, die Bündelung so einzusetzen, dass der Hauptlauf vorrangig über die Schiene abgewickelt wird. Ein Überblick über die Wirkzusammenhänge die in LogoTakt auftreten liefert Abbildung 
3. In dieser Abbildung ist dargestellt, welchen Effekt die Regelmäßigkeit in der Transportdurchführung, der Konsolidierung im Hauptlauf, die Multimodalität im Hauptlauf und die Transporthäufigkeit auf die Kostensenkung im Transport, die Einsparung an Straßenkilometern und Bestandskosten haben.

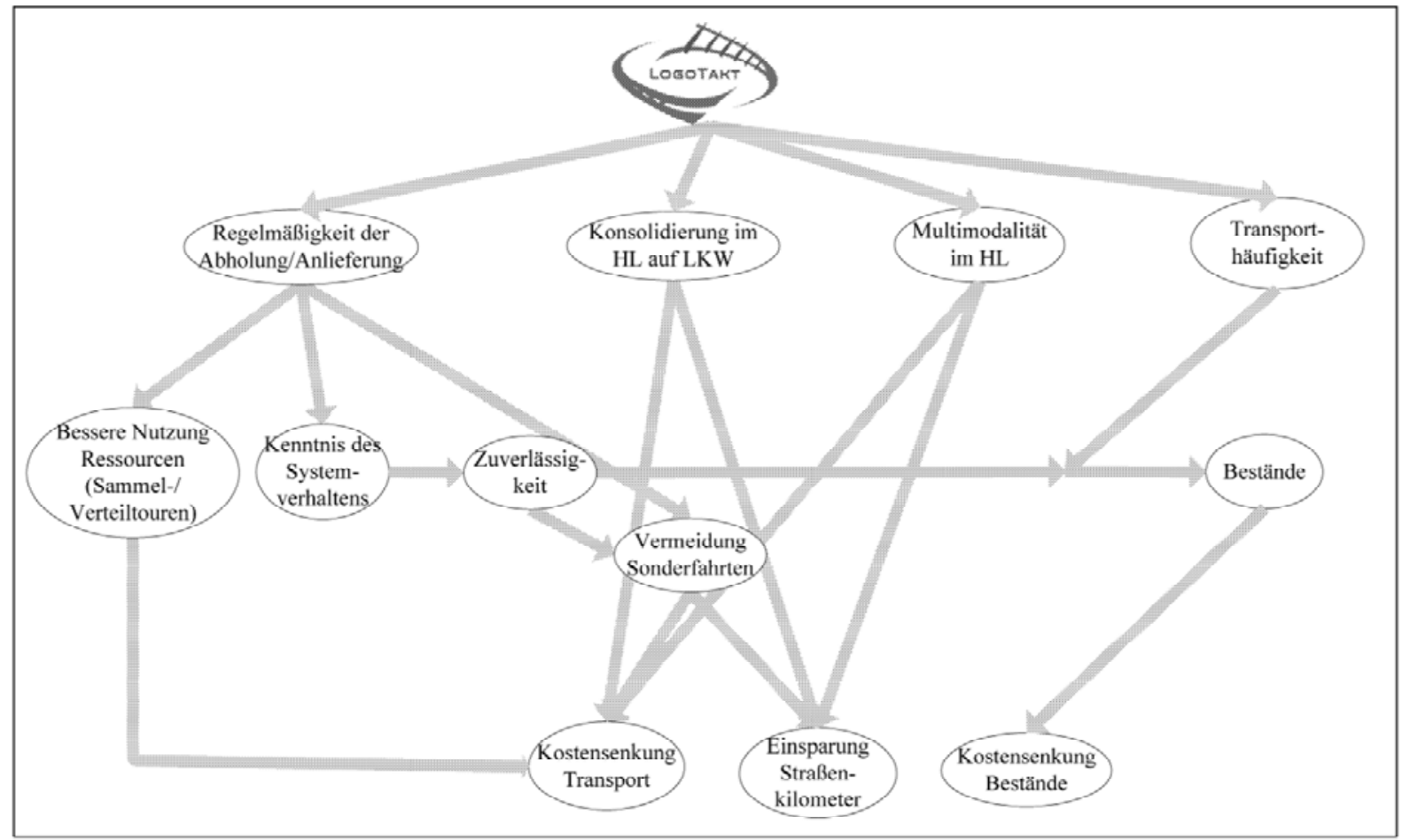

Abbildung 3 Wirkungszusammenhänge des Konzepts LogoTakt; Quelle: [Hin09]

Zusammenfassend handelt es sich bei LogoTakt um ein IT-gesteuertes, flusslogistisches Transportsystem. In diesem Transportsystem spielen notwendigerweise Umschlagvorgänge eine große Rolle. LogoTakt entwickelte daher Instrumente, die die flussorientierte Planung eines Logistiknetzwerkes ermöglichen und mit der Ausführungsebene verbinden, um eine reibungslose Transportdurchführung sicherzustellen.

\section{Planungsebene}

Bündelung und hohe Taktfrequenz machen das Netzwerk komplexer, deshalb potenziell störungsanfälliger, und Umschlagkosten müssen berücksichtigt werden. Aus diesem Grund wurden in LogoTakt Werkzeuge entwickelt, die die Planung der Abholfrequenz ermöglichen, aus den gewünschten Abholfrequenzen effiziente Vorläufe, Hauptläufe und Nachläufe konstruieren und dabei die notwendigen Reservekapazitäten unter Berücksichtigung der Kompensation von Störfällen minimieren. Gleichzeitig ist für den Ist-Zustand anzunehmen, dass die Abrufe pro Zulieferer und in Summe pro Region nicht vollständig nivelliert sein werden. Um dennoch eine akzeptable Auslastung der Transporte zu erreichen, werden verschiedene Instrumente eingesetzt.

Auf Basis kundenindividueller Taktwünsche oder unter Verwendung der Taktplanungskomponente ermittelter Optimaltakte (Optimalität u.a. in Bezug auf 
Transport- und Bestandskosten) wird ein ressourceneffizienter Transportplan erstellt. $\mathrm{Da}$ die Planung in einem offenen Netz erfolgt, können Konsolidierungen und Ausgleiche über verschiedene Branchen hinweg vorgenommen werden, die eine optimale Auslastung der Transportressourcen sicherstellen. Durch diese Konsolidierungen kann eine hohe Transportfrequenz bei gleichzeitiger Wirtschaftlichkeit der Transporte erreicht werden.

\section{Ausführungsebene}

Die Ausführungsebene stellt die Transportdurchführung der Aufträge aus der Planungsebene sicher und nimmt zusätzliche nicht planbare Aufträge auf, die entweder automatisch den geplanten Touren hinzugefügt oder individuell disponiert werden.

Zur Transportlaufzeit werden Statusinformationen über die Transporte an die Disposition von LogoTakt weitergegeben, sodass a) jederzeit ein Abarbeitungszustand eines Auftrages vorliegt, b) individuell in Aufträge eingegriffen werden kann, falls es zu Änderungen oder Störungen kommt und c) nach Transportdurchführung eine Auswertung über die Qualität der Auftragsdurchführung vorgenommen werden kann. Diese Laufzeitinformationen werden gezielt im Verbesserungsmanagement ausgewertet und fließen in die Planung zukünftiger Transporte und die Identifikation von Verbesserungspotentialen innerhalb des Netzes ein.

LogoTakt verknüpft in der Planung, wie bereits erwähnt, erhöhte Abholfrequenzen mit einer netzwerkoptimalen Auslastung der Transportressourcen und führt so zu einer Senkung der Gesamtkosten. Die Kalkulation der netzoptimalen Preise erfolgt gemeinsam mit einem preisgesteuerten Anreizsystem, das Akzente in Richtung einer für alle Netzteilnehmer verbindlichen Transportdurchführung setzt. Die explizite Kalkulation getakteter und damit planbarer Touren kann zu einem Wechsel des Preisschemas von Frachtraten der Gebietsspeditionen in das Preisschema von Teilladungen überführt werden, diese sind tendenziell günstiger.

Die Robustheit in der Transportdurchführung wird neben der systeminhärent hohen Transportfrequenz über ein Abweichungs- und Störungsmanagement zur Laufzeit erreicht, das rechnergestützt Lösungsvorschläge auf unterschiedlichen Eskalationsebenen bewertet und anbietet (vgl. Abbildung 4, [Pul10b]). Dargestellt sind die Ebenen: Tour, Transport, Auftrag, Flotte und der LogoTakt-Verbund (z.B. die am System beteiligten Speditionen), sowie den Handlungsmöglichkeiten je Ebene. Bei der Lösungssuche wird immer auf der untersten Ebene, der Tour-Ebene, begonnen. Dadurch ist sichergestellt, dass möglichst geringe Eingriffe in geplante Fahrpläne vorgenommen werden müssen bzw. möglichst wenige Abhängigkeiten beachtet werden müssen.

Darüber hinaus wird ein automatisiertes Verbesserungsmanagement auf der Planungsseite des Netzes zur Verfügung gestellt, in dem erfolgreiche Handlungsmuster aus der Vergangenheit in die zukünftige Gestaltung von 
Transporten und des Netzwerkes einfließen. Weiterhin wird die Robustheit auf Planungsebene durch ein zeitdiskretes Routing im Netzwerk erhöht, indem auftragsbezogene Zeitpunkte des Netzwerkübergangs ermittelt werden.

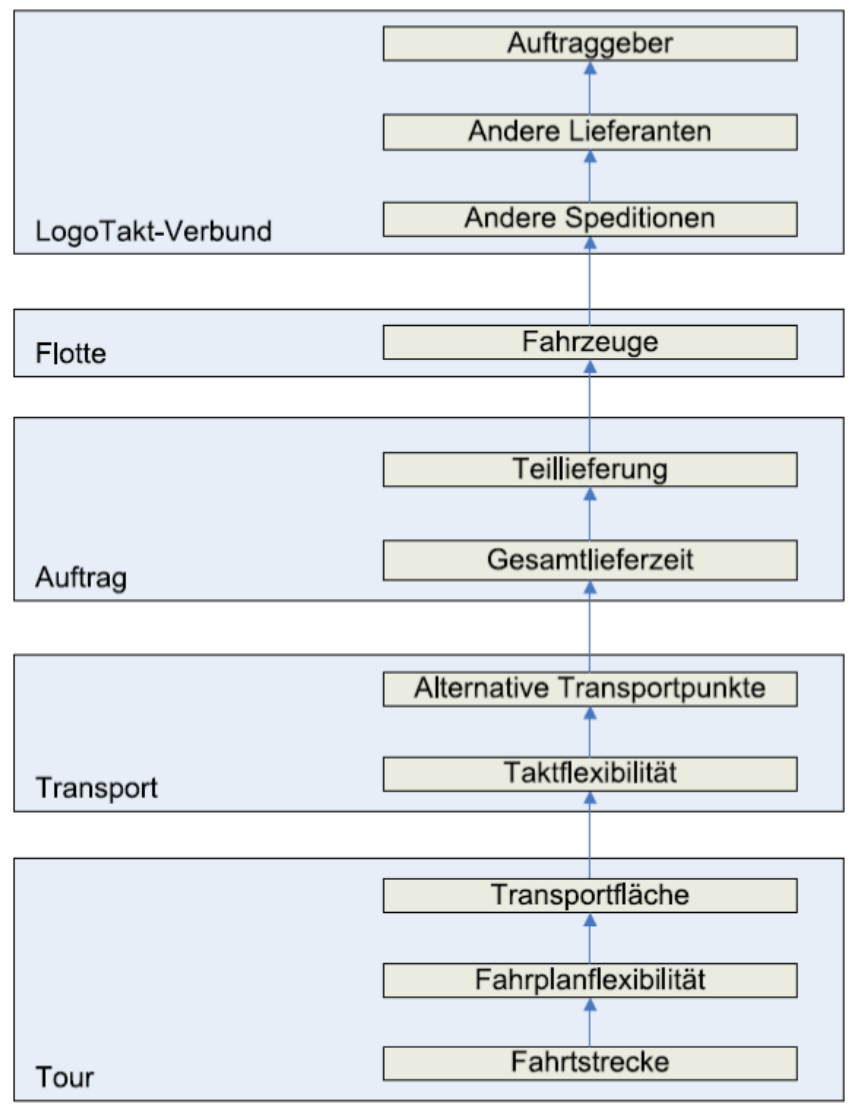

Abbildung 4 Eskalation des automatisierten Abweichungsmanagements über Lösungsebenen; Quelle: [Pul10a]

\section{Ergebnisse}

Im Rahmen des Projektes, dessen Laufzeit bis Ende 2010 reicht, werden derzeit Simulationsrechnungen durchgeführt und ein Pilotbetrieb umgesetzt, um den Einfluss hochfrequenter kleinteiliger Sendungen auf Wirtschaftlichkeit und die Belastung des Straßenverkehrsnetzes zu quantifizieren.

\subsection{Simulation}

Zum Zeitpunkt der Einreichung des Beitrages liegen bereits erste Ergebnisse der szenarienbasierten Simulation vor, die im Folgenden besprochen werden sollen. Die Simulationen werden bis Ende $2010 \mathrm{zu}$ folgenden Bereichen durchgeführt: Wirtschaftlichkeit des Systems bei schwankendem Abrufverhalten von Transporten, Wirtschaftlichkeit in Abhängigkeit der Taktfrequenzen je Verlader und Region, Wirtschaftlichkeit bei kooperativem Verhalten am Beispiel der Automobilindustrie.

Als Basis der Wirtschaftlichkeitsanalyse bei schwankendem Abrufverhalten dienen volkswirtschafliche Daten mit Sendern und Empfängern, deren Sendungsstruktur als Aufkommen für das Transportkonzept LogoTakt geeignet ist (vgl. [Bre09]). Betrachtet man die räumliche Verteilung dieser Sender und Empfänger kann festgestellt (c) 2010 Logistics Journal : Proceedings - ISSN 2192-9084 
werden, dass die Schwerpunkte in den Wirtschaftsregionen Süddeutschland und an der Rheinschiene liegen (vgl. Abbildung 5).
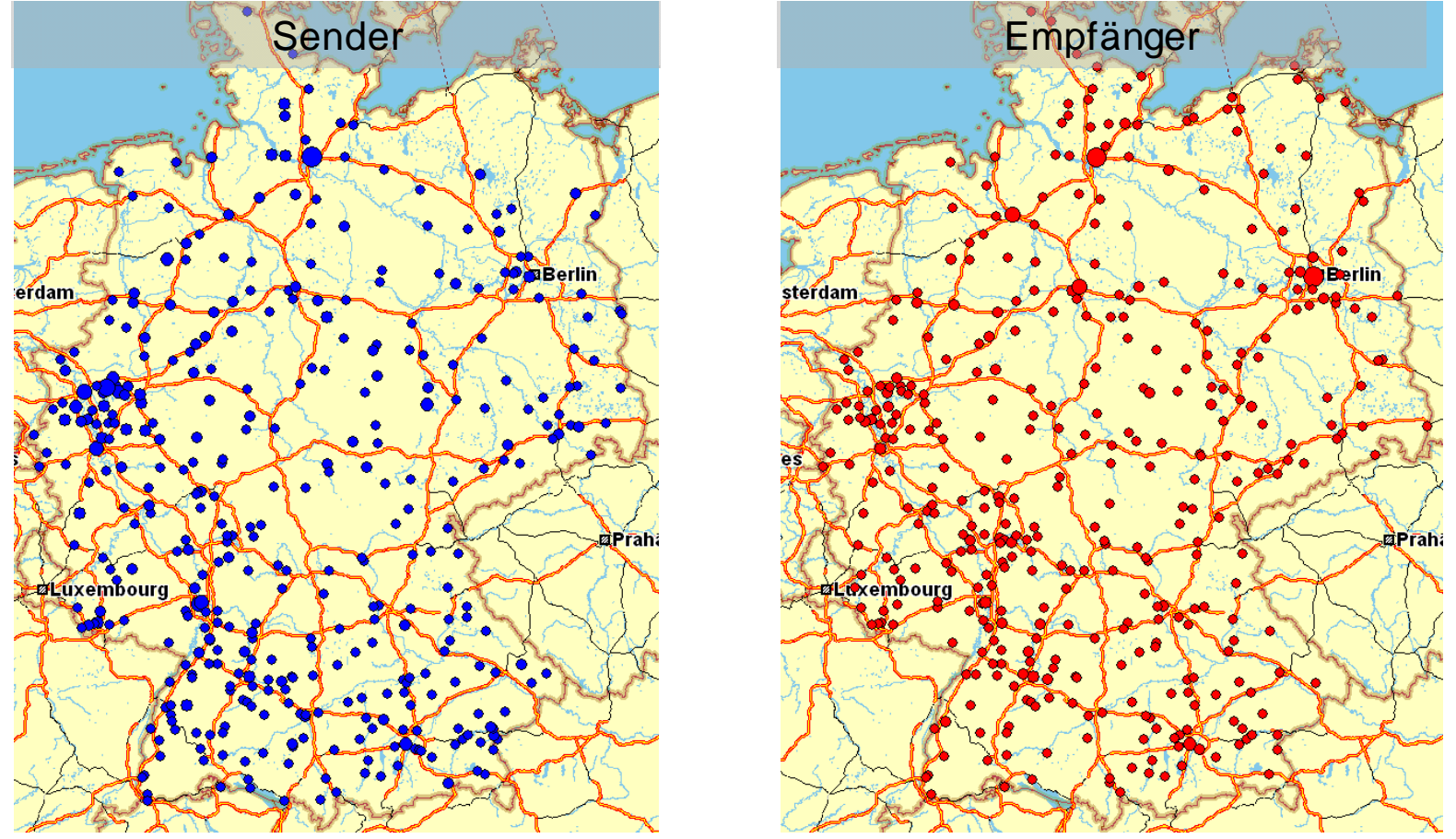

Abbildung 5 volkswirtschaftliches LogoTakt-Aufkommen in Deutschland, Quelle: Locom

In ersten Auswertungen konnte eine gleichmäßigere Belastung in den Umschlagspunkten festgestellt werden. Diese sind darauf zurückzuführen, dass Sender ihre Transporte nicht mehr konsolidieren müssen und höherfrequenter versenden können, wodurch gleichmäßigere Abrufe und damit eine gleichmäßigere Auslastung entstehen. Durch die Harmonisierung des Aufkommens in Vor- und Nachläufen ist auch eine gleichmäßigere Belastung über die Wochentage und dadurch eine Reduktion von Schwankungen in der Auslastung, um ungefähr sieben Prozent, festzustellen.

Vergleicht man die Transportkosten, die pro Palette entstehen, kann man zwischen nivelliertem und schwankendem Aufkommen nur geringe Unterschiede feststellen, die unter anderem davon abhängen, wie gut die Auslastung der Waggons im Hauptlauf ist. Dabei ist jedoch noch nicht berücksichtigt, dass die Bestände aufseiten der Sender und Empfänger, aufgrund der hochfrequenteren Abholung kleiner ausfallen, und ebenfalls monetär bewertet werden müssten (vgl. [Sel09]). Weiterhin führt die gleichmäßigere Auslastung von Transport- und Umschlagsressourcen ebenfalls zu weniger gebundenem Kapital, da weniger Kapazitäten vorgehalten werden müssen.

Im Projekt LogoTakt können durch den Einsatz der Bahn im Hauptlauf im Schnitt über den Transport von Versender zu Empfänger ungefähr 30 Prozent der Straßenverkehrskilometer eingespart werden. Gleichzeitig ergibt sich durch die Verlagerung des Verkehrs eine Einsparung der $\mathrm{CO}^{2}$-Emissionen. 
Weitere positive Effekte können durch die Kooperation von Unternehmen erzielt werden. In diesen Simulationen wird die Automobilindustrie in Deutschland basierend auf Hochrechnungen näher untersucht. Durch die Kooperation der Automobilhersteller und ihrer Zulieferer der ersten Stufe in der Transportabwicklung können die Transportkosten bei Einsatz des LogoTakt Konzeptes um ca. zwei Prozent gesenkt werden. Durch die Ausnutzung der räumlichen Nähe, vor allem der Zulieferer, sind auch hier Einsparungen in Bezug auf die Straßenverkehrskilometer zu erwarten.

\subsection{Pilotbetrieb}

Der Pilotbetrieb wurde Mitte 2010 aufgenommen. Innerhalb eines Distributionssystems mit Sendern in Deutschland und Empfängern in Italien wird die Belieferung von einmal pro Woche mit Lkws stufenweise auf mehrmals pro Woche im intermodalen Verkehr umgestellt. Ziel des Piloten ist es, die praktische Umsetzung des Konzeptes zu demonstrieren sowie Ergebnisse mithilfe des realen Betriebs zu validieren.

Die Touren für den Pilotbetrieb werden auf Basis von Abrufdaten einen Monat im Voraus strategisch geplant. Drei Tage vor Versandtermin wird die aktuelle Planung, mit eventuellen Abweichungen, an das System übermittelt. Weiterhin werden Transportdaten zu Staus, Verspätungen, etc. in das System eingepflegt, um das Abweichungsmanagement auf seine Funktionsfähigkeit sowie die Qualität der Lösungsvorschläge zu überprüfen. Anschließend werden diese in einer Historiedatenbank gespeicherten Daten durch das Verbesserungsmanagement analysiert, wodurch beispielsweise die Planung der Mengenpuffer überprüft und gegebenenfalls angepasst werden kann.

Durch die Erhöhung der Transportfrequenz, derzeit von einmal auf zweimal wöchentlich, können kurzfristige Bestellungen, die bisher teilweise durch Sondertransporte abgewickelt werden mussten, im Rahmen der geplanten Transporte mitberücksichtigt werden. Hierin zeigt sich der Vorteil der höheren Transportfrequenz im Vergleich zu herkömmlichen Transportverfahren, die Waren zunächst ansammeln, um dann gebündelt zu transportieren. LogoTakt ist ein System, das eine konsolidierte Planung über unterschiedliche Unternehmen hinweg vornehmen kann. Damit wird es möglich kleinere Sendungsmengen wirtschaftlich und häufiger zu transportieren.

\section{$3 \quad$ Zusammenfassung}

Das Transportsystem LogoTakt stellt ein innovatives System zum hochfrequenten, intermodalen Transport dar. Erste Ergebnisse aus Simulation und Praxisbetrieb versprechen positive Effekte in Bezug auf die Reduktion der Straßenverkehrsbelastung und $\mathrm{CO}^{2}$-Emissionen, aber auch bezüglich Kapitalbindungskosten von Ressourcen und Beständen. Diese Effekte sind im weiteren Verlauf des Projektes quantitativ zu bewerten, um festzustellen, ob ein (c) 2010 Logistics Journal : Proceedings - ISSN 2192-9084 
solches System Vorteile bietet und eine breite Umsetzung angestoßen werden sollte. Um dieses Konzept erfolgreich auszuweiten, müssten gleichzeitig Logistikknoten auf operativer Ebene ausgebaut werden, um einen wirtschaftichen Umschlag von Teilpartien im Großladungsträgerbereich zu ermöglichen. Derzeit werden aufgrund dieser Problematik intermodale Verkehre, die für die Entlastung des Straßennetzes von hoher Bedeutung sind, von produzierenden Unternehmen kaum eingesetzt, obwohl sie bei ausreichend hoher Taktfrequenz die Ziele der Produktionslogistik sehr gut stützen könnten. Andererseits zeigt der effiziente Betrieb von Kurier- und Expressdiensten im Bereich der Kleinladung, dass mit den entsprechenden intralogistischen Einrichtungen ein kostengünstiger Umschlag von Paketen möglich ist. 


\section{Literatur}

[Bre09] Breier, Heiko; Liedtke, Gernot; Walther, Christoph; Furmans, Kai: LogoTakt - Realisierung robuster getakteter Verkehre in intermodalen Transportnetzwerken; in Höchstleistung im spurgeführten System: Welche Potenziale und Innovationen gibt es noch im Güter-, Nah- und Fernverkehr?. Dresden: 22. Verkehrswissenschaftliche Tage, 2009.

[Bun10] Bundesamt für Güterverkehr: BAG-Mautstatistik: Ergebnisse für das 1. Halbjahr 2010, Laufende Nr. 15/10. Köln: 2010.

[Car08] Cardeneo, A.; van Dinther, C.; Furmans, K.; Weinhardt, C.; Inderfurth, K.; Neumann, G.; Schenk, M.; Wäscher, G. und Ziems, D.: Offene getaktete robuste multimodale Dienstleistungsnetze. Magdeburg: Netzwerklogistik - 13. Magdeburger Logistik-Tagung, 2008.

[Fur10] Furmans, Kai; Breier, Heiko, Weiblen, Judith: Forschungsprojekt LogoTakt: hochfrequente Anlieferung kleiner Ladungen (S. 54-55). In: Karlsruher Wirtschaftsspiegel 2010/2011. Karlsruhe: Stadt Karlsruhe Wirtschaftsförderung, 2010.

[Hin09] Hinding, Melanie; Heckmann, Iris; Furmans, Kai: LogoTakt - ein offener, getakteter und robuster Transportprozess(S. 219-232); in Nachhaltigkeit in flexiblen Produktions- und Liefernetzwerken. Münster: MV-Verlag, 2009.

[Oel09] Oelbermann, Anne; Breier, Heiko: Offene Transportnetze Kollaborative Transportplanung unter Nutzung eines Taktes (S. 293305); in Nachhaltigkeit in flexiblen Produktions- und Liefernetzwerken. Münster: MV-Verlag, 2009.

[Pul10a] Pulter, Natalja; Nimis, Jens; Lockemann, Peter: Störungsmanagement in offenen, getakteten Logistiknetzwerken (S.131-135); in KI-Künstliche Intelligenz. Berlin: Springer, 2010.

[Pul10b] Pulter, Natalja; Nimis, Jens; Lockemann, Peter: Managing Contigencies in Timed Transportation Networks by Agent Technology, Workshop Proceedings of the Artificial Intellegence and Logistics Workshop at ECAI 2010. Lisbon: 2010.

[Rat10] Ratzinger, Ralf: Gleitende Mittelfristprognose für den Güter- und Personenverkehr- Kurzfristprognose Sommer 2010 im Auftrag des Bundesministeriums für Verkehr, Bau und Stadtentwicklung FE-Nr. 96.0927/2009. München: 2010.

[Sel08] Selinger, Ulrich; Liedtke, Gernot; Hinding, Melanie: High frequency delivery for medium flow of goods; in Conference Proceedings of the International Trade and Freight Transportation Conference ITFTC. Cyprus, 2008. 